\title{
Análises de cumarinas em derivados de citrus utilizando cromatografia gasosa de alta resolução com sistemas de detecção por ionização de chama e espectrometria de massas
}

\section{Analysis of coumarin derivatives of citrus using high resolution gas chromatography with detection systems flame ionization mass spectrometry}

\author{
Paulo César Cavalcante Vila Nova ${ }^{1 *}$, Janete Harumi Yariwake Vilegas ${ }^{2}$, Fernando Mauro \\ Lanças $^{2}$, Eduardo José de Arruda ${ }^{3}$ e Lincoln Carlos Silva de Oliveira ${ }^{1}$ \\ ${ }^{1}$ Departamento de Química, Universidade Federal de Mato Grosso do Sul, 79074-460 Campo Grande-MS, Brasil. \\ ${ }^{2}$ Instituto de Química de São Carlos, Universidade de São Paulo, 13560-970 São Carlos-SP, Brasil. ${ }^{3}$ Faculdade de \\ Ciências Exatas e Tecnologia/Química, 79804-970, Dourados-MS, Brasil.
}

\begin{abstract}
Citric plants, specially orange, are of great importance in Brazilian economy, mainly Southeast region. The essential oils from Citrus are one of the most important of raw material group of several industries, mainly for foods, pharmaceutical and toiletry. In foods industry, orange essential oil is utilized to enhance the juice flavor and it is exported, more specific to United States (main consumer). In this work was developed an analytical methodology for identification and quantification of coumarins in citric plants (oils, wax) using chromatographic techniques such as high resolution gas chromatography with flame ionization detector (HRGC-FID) and mass spectrometry detector (HRGC-MS). The results of HRGC-MS qualitative analyses using selective ion monitoring (SIM) indicated the presence of coumarin in the samples of essential oil and wax, and in the latter it was detected the presence of coumarin and psoralen. Quantitative analyses by external standard methods, internal standard and addition of standard showed good results, specially internal standard method which showed greater repeatebility were statistically identical ( $t$ test and ANOVA, $p=0.95$ ).
\end{abstract}

Key-words: Coumarins, Citrus derivatives, HRGC-MSD

\section{INTRODUÇÃO}

Os óleos essenciais são usados principalmente para realçar o sabor e aroma dos sucos de laranja processados. Têm relação direta com a qualidade dos produtos aos quais eles são adicionados. Devido à grande importância dos óleos cítricos, a grande maioria produzida no Brasil é exportada para os EUA (Simões e SPitzer, 1999; Kuster e Roche, 2001). São usados como matéria-prima de grande importância para várias indústrias, principalmente alimentícia, farmacêutica e de perfumaria.

Além do uso do óleo essencial de laranja para melhorar a qualidade do "flavor" nos sucos, têmse aproveitado do óleo os seus constituintes tais como o citral e o citronelal para uso em fragrâncias, sabões, detergentes, cremes, loção e perfumes, bebidas não alcoólicas, sorvetes, doces, gelatinas, xaropes, goma de mascar, solventes e intermediários de síntese A Associação Internacional de Fragrância limita a quantidade de óleo de limão a $4 \%$ em loção e perfume (Biavatte et. al, 2005; Tanaka, 2005, Swingle e Reece, 2005).

Em se tratando dos compostos de análise, as cumarinas tem uma grande aplicação industrial, devido principalmente à sua forte fragrância. É utilizada em adoçantes, lavandas, perfumes, aditivos de alimentos em combinação com a vanilina, aditivos de tintas e de borrachas para mascarar o odor e como estabilizador de sabor/odor de tabacos (De Miranda, 2009). As

Author for correspondence: vilanovapc@gmail.com

J. Biotec. Biodivers. v. 3, N.1: pp. 25-31, Fev. 2012 
furanocumarinas, especialmente o psoraleno e o bergapteno, são utilizadas como fotodinamizadores em bronzeadores, ou seja, aumentam o bronzeamento induzido por radiação ultravioleta; porém, a sua utilização não é isenta de riscos, podendo ocorrer à aparição de dermatites.

Neste trabalho desenvolveu-se uma metodologia analítica de identificação e quantificação por cromatografia gasosa para identificação e quantificação dos compostos cumarina, psoraleno e bergapteno em amostras de óleo essencial e cera de laranja e comparou-se a extração por prensagem a frio (FMC) usada regularmente nas indústrias de suco com a extração convencional por arraste a vapor.

$\mathrm{Na}$ análise qualitativa, foi identificado nas amostras de óleo somente a cumarina e na amostra de cera foram encontradas a cumarina (figura 1) e o psoraleno (figura 2). Os resultados quantitativos utilizando os métodos do padrão externo, padrão interno e adição de padrão foram avaliados e verificou-se que o método do padrão interno apresentou maior repetibilidade. Verificou-se que a extração por arraste a vapor pode ser utilizado na análise destas cumarinas, obtendo-se resultados semelhantes à extração por prensagem a frio.<smiles>O=c1ccc2ccccc2o1</smiles>

Figura 1 - Cumarina
Figura 2 - Psoraleno<smiles>O=c1ccc2cc3ccoc3cc2o1</smiles>

\section{MATERIAL E MÉTODOS Padrões}

Foram analisadas soluções-padrão de cumarina, psoraleno e bergapteno (todos Sigma e Aldrich) em diferentes concentrações, na faixa de 1,0 a $0,0001 \mathrm{mg} / \mathrm{mL}$ usando metanol como solvente.

\section{Amostras}

As amostras de cera e óleo essencial de laranja utilizadas em todas as análises foram obtidas por prensagem a frio (FMC) e fornecidas pelas indústrias da região (amostras I, II e III) e por arraste a vapor (amostra IV). Estas diferentes amostras foram obtidas em épocas distintas e para diferenciação foram denominadas da seguinte maneira:

Óleo I e cera: obtido da indústria Citrosuco e fornecidos pelo Prof. Dr. José Vladimir de Oliveira, da UFRJ, em meados de agosto de 1997.
A cera provém do óleo essencial e é separado por centrifugação. A amostra foi estocada em geladeira a $5^{\circ} \mathrm{C}$ até a data de análise.

Óleo II: fornecido pelo Sr. Wilson Andalécio, obtido da indústria de suco Cutrale, coletado no tanque de armazenamento em 06/08/99 e estocada a $5^{\circ} \mathrm{C}$ na geladeira.

Óleo III: Também fornecido pelo Sr. Wilson Andalécio, obtido da indústria de suco Cutrale, coletado na centrífuga em 27/08/99. A amostra foi estocada a $5^{\circ} \mathrm{C}$ na geladeira.

Óleo IV: Extraído no laboratório do IQSC, por arraste a vapor a partir de cascas de laranja em 02/09/99 e estocada na geladeira a $5^{0} \mathrm{C}$.

\section{Preparação das amostras}

Para obtenção do óleo essencial (IV), foram utilizadas laranjas do tipo pêra adquiridos no comércio da cidade de São Carlos-SP, descascadas manualmente e a polpa foi separada e triturada em um processador de alimentos. O óleo foi extraído utilizando um sistema de arraste a vapor. Foi realizado uma partição adicionando $50 \mathrm{~mL}$ de nhexano, formando duas fases, sendo que na fase hexânica obtida, foi adicionado $50 \mathrm{~g}$ de Sulfato de Sódio anidro, com a formação de duas fases. A fase hexânica, contendo o óleo essencial, foi utilizada para injeção nos cromatógrafos.

Os demais óleos (I, II e III), fornecidos pelas indústrias de suco de laranja do Estado de São Paulo, obtidos por prensagem a frio (FMC). O óleo I foi processado na Indústria Citrosuco (Matão, SP), em Agosto de 1997. O óleo II e III foram processados nas Indústrias de suco Cutrale (Araraquara, SP), em Agosto de 1999. Todas as amostras foram armazenadas a $5^{0} \mathrm{C}$ para posterior análise e foram submetidos à centrifugação a 5000rpm para separação da fase oleosa e fase sólida (cera). A fase óleo foi filtrada em algodão e preparada dissolvendo $0,03 \mathrm{~g} / 10 \mathrm{~mL}$ de $\mathrm{n}$-hexano. Foi preparada uma mistura de $0,03 \mathrm{~g}$ de cera $/ 10 \mathrm{~mL}$ de n-hexano para posterior injeção nos cromatógrafos.

\section{Condições Cromatográficas}

Cromatografia Gasosa de Alta ResoluçãoDetecção por fotoionização

As cumarinas foram analisadas em triplicata, após injeção de $1 \mu \mathrm{L}$ da fase orgânica em cromatógrafo a gás SRI 8610 (SRI Instruments, Torrence, USA), acoplado ao detector PID com lâmpada UV de $121,6 \mathrm{~nm}$, utilizando coluna capilar LM-5 (5\% fenil $95 \%$ metilpolisiloxano), $22 \mathrm{~m} \times 0,53 \mathrm{~mm}$ d. i. 
x $1,4 \mu \mathrm{m}$ d.f.; gás de arraste: hidrogênio com velocidade linear de $26,2 \mathrm{~cm} / \mathrm{s}$, com programação de temperatura para a cumarina: $230{ }^{\circ} \mathrm{C}(5 \mathrm{~min})$ a $10{ }^{\circ} \mathrm{C} / \mathrm{min}$ até $250{ }^{\circ} \mathrm{C}(15 \mathrm{~min})$, psoraleno: $80{ }^{\circ} \mathrm{C}$ a $10{ }^{\circ} \mathrm{C} / \mathrm{min}$ até $250{ }^{\circ} \mathrm{C}(15 \mathrm{~min})$, usando o tipo de injeção on-column.

Cromatografia Gasosa de Alta ResoluçãoDetecção por Ionização de Chama

As cumarinas foram analisadas em triplicata, após injeção de $1 \mu \mathrm{L}$ da fase orgânica em cromatógrafo a gás da HP 5890 Série II, acoplado ao detector FID, utilizando coluna OV-73 (5\% fenil, 95\% polidimetilsiloxano), $15 \mathrm{~m} \mathrm{x} 0,25 \mathrm{~mm} \times 0,25 \mu \mathrm{m}$, com programação de temperatura $40{ }^{\circ} \mathrm{C}(2 \mathrm{~min}) \mathrm{a}$ $4{ }^{0} \mathrm{C} / \mathrm{min}$ até $130{ }^{0} \mathrm{C}$ a $8{ }^{\circ} \mathrm{C} / \mathrm{min}$ até $300{ }^{\circ} \mathrm{C}$; Temperatura do detector $=310{ }^{\circ} \mathrm{C}$, gás de arraste: hidrogênio, com fluxo de $3 \mathrm{~mL} / \mathrm{min}$. Fluxo dos gases de queima: $40 \mathrm{~mL} / \mathrm{min}\left(\mathrm{H}_{2}\right)$ e $400 \mathrm{~mL} / \mathrm{min}$ (ar sintético), injeção split/splitless (1:9) e temperatura do injetor de $250{ }^{\circ} \mathrm{C}$.

Cromatografia Gasosa de Alta ResoluçãoDetecção por Espectrometria de Massas

As cumarinas foram analisadas em triplicata, após injeção de $1 \mu \mathrm{L}$ da fase orgânica em cromatógrafo HP 5890 acoplado ao espectrômetro de massas MSD HP5970, utilizando coluna LM-5 (5\% fenil $95 \%$ metilpolisiloxano), $55 \mathrm{~m} \times 0,25 \mathrm{~mm} \times 0,35 \mu \mathrm{m}$, programação: $100{ }^{\circ} \mathrm{C}(5 \mathrm{~min})$ a $8{ }^{\circ} \mathrm{C} / \mathrm{min}$ até 250 ${ }^{0} \mathrm{C}$ (20min), razão de split 1:9, hélio como gás de arraste à velocidade linear de $26,8 \mathrm{~cm} / \mathrm{s}$; a detecção no MS: EI, $70 \mathrm{eV}$, modo SCAN: 45 a 475 u.m.a .; modo SIM:

$\checkmark$ Íons monitorados da cumarina: m/z 90, m/z 118 e m/z 146;

$\checkmark$ Íons monitorados do psoraleno: $\mathrm{m} / \mathrm{z}$ 130, m/z 158 e m/z 186;

$\checkmark$ Íons monitorados do bergapteno: $\mathrm{m} / \mathrm{z} 173, \mathrm{~m} / \mathrm{z}$ 201 e m/z 216.

\section{RESULTADOS E DISCUSSÕES Análises por HRGC-FID}

As figuras 3 e 4 mostram os cromatogramas referentes às diferentes amostras do óleo essencial de laranja obtido por prensagem a frio e por arraste a vapor e a figura 4 mostra o cromatograma referente à amostra de cera obtida por prensagem a frio. A identificação dos compostos analisados encontram-se especificada nos cromatogramas. Nas figuras 3 a 5 , pode-se verificar que os perfis cromatográficos das amostras de óleo e cera são bem semelhantes, os picos estão bem separados e que nas amostras de óleo constata-se preliminarmente somente a presença de cumarina, enquanto que na amostra de cera pode-se verificar a presença de cumarina e psoraleno. Em todas as amostras a presença de bergapteno não foi detectada. Estes cromatogramas caracterizam-se por vários picos intensos com tr pequeno e poucos picos de maior tr. Os picos mais intensos são os hidrocarbonetos terpênicos, sendo que o dlimoneno é o mais abundante (Robards e Antolovich, 1995; Moshonas e Shaw,1994).

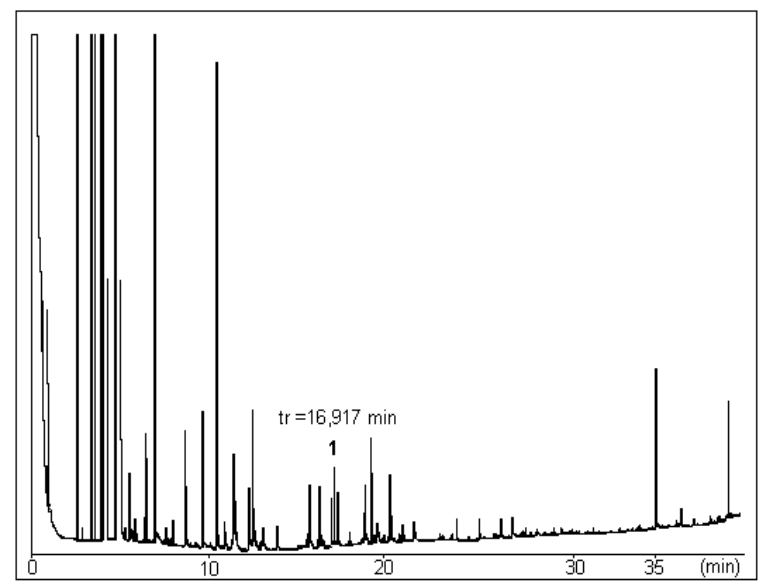

Figura 3 - Cromatograma obtido por HRGC-FID da amostra de óleo I obtido por prensagem a frio. (1): cumarina.

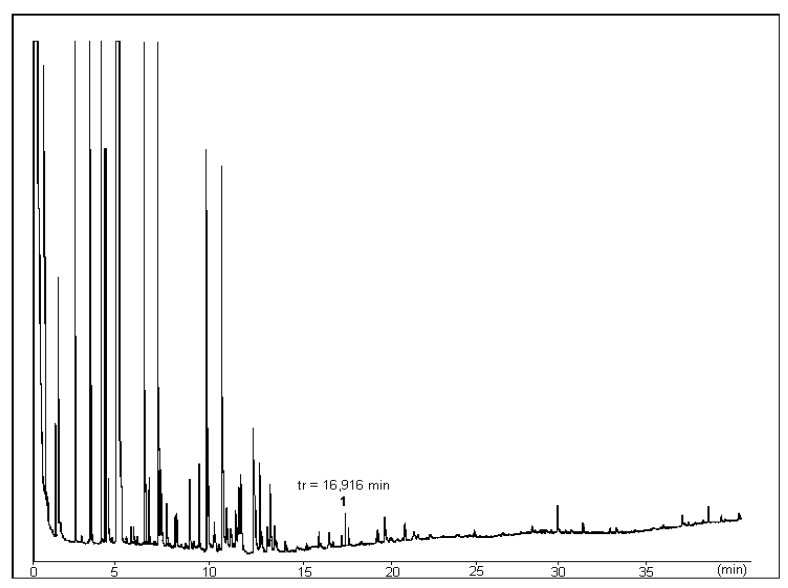

Figura 4 - Cromatograma obtido por HRGC-FID da amostra de óleo II obtido por arraste a vapor. (1): cumarina. 


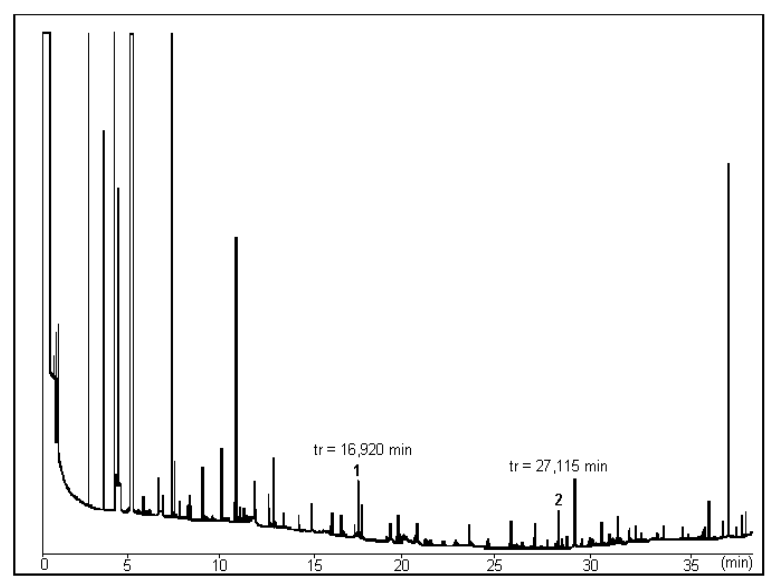

Figura 5 - Cromatograma obtido por HRGC-FID da amostra de cera obtida por prensagem a frio. (1): cumarina, (2): psoraleno.

\section{Análises por HRGC-MS}

Os compostos analisados foram a cumarina, o psoraleno e o bergapteno. Foram injetados os padrões no HRGC-MSD no modo SCAN e os espectros de massas obtidos foram comparados com aqueles da biblioteca NBS (Figuras 6 e 7), com o Eight Peak Index e com a literatura. Foi encontrado um bom nivel de concordância entre os fragmentos obtidos para cada composto neste trabalho (Eight Peak Index of Mass Spectra, 1986). Os resultados das análises por HRGC-MS para verificação da autenticidade dos padrões serviram de base para a escolha dos íons de cada composto visando o estudo da presença de cumarinas nas amostras de óleo essencial de Citrus e esta confirmação (identificação) foi realizada através do monitoramento do íon selecionado (SIM) e comparando-se o tempo de retenção dos íons selecionados com cada padrão injetado.

$\mathrm{Na}$ Tabela 1 encontra-se um resumo dos resultados da análise qualitativa das diferentes amostras de óleo essencial e cera utilizando a espectrometria de massas, no modo SIM.

Tabela 1. Resultados da análise qualitativa (HRGC-MS, modo SIM) de cumarina, psoraleno e bergapteno nas diferentes amostras de óleo essencial e cera.

\begin{tabular}{cccc} 
Amostras & cumarina & psoraleno & bergapteno \\
\hline Óleo I & + & - & - \\
Cera & + & + & - \\
Óleo II & + & - & - \\
Óleo III & + & - & - \\
Óleo IV & + & - & - \\
\hline
\end{tabular}

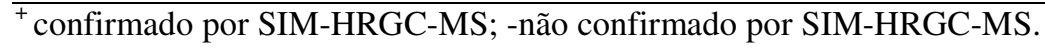

Os resultados das análises por HRGC-MS para verificação da autenticidade dos padrões serviram de base para a escolha dos íons de cada composto visando o estudo da presença de cumarinas nas amostras de óleo essencial de Citrus e esta confirmação (identificação) foi realizada através do monitoramento do íon selecionado (SIM) e comparando-se o tempo de retenção dos íons selecionados com cada padrão injetado (Vilegas, 1997; Loghkin e Sckanyan, 2006).

A Figura 6 mostra o TIC e o espectro de massas obtido na análise no modo SIM para a amostra do óleo I, confirmando-se a presença de cumarina ( $\operatorname{tr}=20,607 \mathrm{~min}$ ). Na Figura 7, pode-se observar o cromatograma do íon total e o fragmentograma obtido na análise de amostra de cera no modo SIM confirmando-se a presença de cumarina (tr=20,696min) na referida amostra (Pathak e Fitzpatrick, 1992).

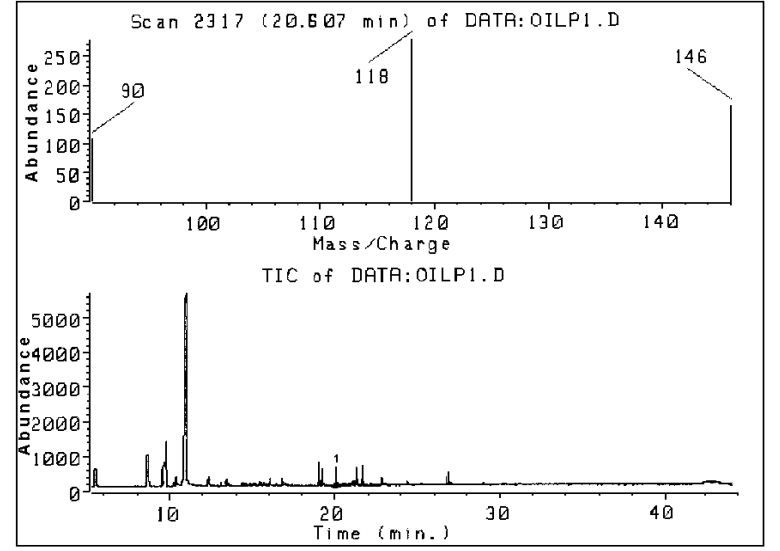

Figura 6 - Cromatograma do íon total do óleo I obtido na análise no modo SIM (m/z 90, m/z 118 e m/z 146) para o monitoramento do composto cumarina (1), $\operatorname{tr}=20,607 \mathrm{~min}$. EI, 70eV. 


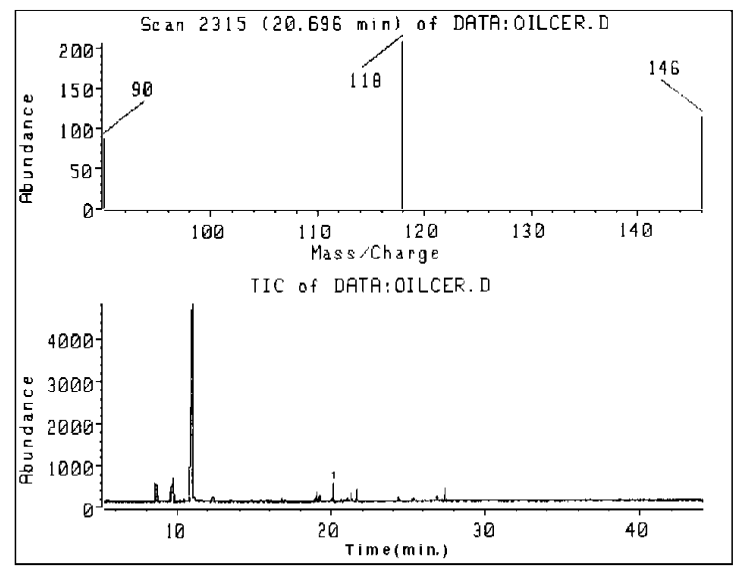

Figura 7 - Cromatograma do íon total da amostra de cera de laranja na análise no modo SIM (m/z 90, m/z 118 e $\mathrm{m} / \mathrm{z}$ 146) para o monitoramento da cumarina (1), $\operatorname{tr}=20,696 \mathrm{~min}$. EI, 70eV.

\section{Análises Quantitativas}

A cumarina presente nas diferentes amostras de óleo essencial extraído por prensagem a frio (FMC) ou por arraste a vapor realizado no laboratório (óleo I a IV) e o psoraleno e a cumarina presentes na amostra de cera, foram quantificados por HRGC-FID empregando-se os métodos do padrão externo e do padrão interno. Somente para as amostras de óleo I e de cera foi aplicado o método da adição de padrão, de acordo com a Tabela 2. Esses resultados foram analisados estatisticamente de acordo com o teste t e ANOVA e os resultados indicaram que os dados são estatisticamente iguais com 95\% de confiança (Microcal, 1996; Ribani, 2004).

Tabela 2. Concentração ${ }^{a}$ de cumarinas $(\mathrm{mg} / \mathrm{mL})$ em amostras de derivados de Citrus, empregando HRGCFID.

\begin{tabular}{cccc}
\hline Amostras & Padrão externo $^{\mathbf{b}}$ & Padrão interno $^{\mathbf{c}}$ & Adição de padrão $^{\text {Páleo I }}$ \\
\hline Óleo II & $0.00312 \pm 0,00006$ & $0.00313 \pm 0.0000100$ & $0.002880 \pm 0.000107$ \\
Óleo III & $0.00340 \pm 0.00002$ & $0.00357 \pm 0.0000387$ & - \\
Óleo IV & $0.00363 \pm 0.00004$ & $0.00335 \pm 0.0000240$ & - \\
Cera & $0.00311 \pm 0.00002$ & $0.00350 \pm 0.0000141$ & - \\
\hline
\end{tabular}

${ }^{\mathrm{a}}$ (média \pm desvio padrão); mg cumarina / $\mathrm{mL}$ de amostra; ${ }^{\mathrm{b}, \mathrm{c}}$ Resultados estatisticamente iguais (ANOVA e teste $\mathrm{t}, p=$ $0.95)$.

Comparando-se com os resultados da Tabela 2, observa-se que os resultados das análises pelo método da adição de padrão foram semelhantes aos obtidos pela quantificação pelo método do padrão externo. Mas, ao se observar os intervalos de confiança e os valores de desvio padrão obtidos em ambos os métodos, podemos considerar a quantificação pelo método da adição de padrão como tendo menor repetibilidade. Esses dados também sugerem que a concentração de cumarinas não são afetadas na estocagem do óleo essencial, obtida por prensagem a frio (processados entre 1997 e 1999), dando resultados estatisticamente idênticos comparados com outras amostras de óleo (Ribani, 2004).
A Tabela 3 apresenta os resultados da determinação da concentração dos dois compostos (cumarina e psoraleno) presentes nos óleos e cera de Citrus, obtidos em diferentes épocas e processos. Esses resultados foram analisados estatisticamente de acordo com $\mathrm{o}$ teste $\mathrm{t} e$ ANOVA, e os resultados indicaram que os dados são estatisticamente iguais com $95 \%$ de confiança (Microcal, 1996). 


\begin{tabular}{|c|c|c|c|c|}
\hline I Amostras & $\begin{array}{l}\text { Concentração média } \\
(\mathrm{mg} / \mathrm{mL}) \text { da cumarina }\end{array}$ & $\begin{array}{l}\text { SD }^{1} \\
(+/-)\end{array}$ & $\begin{array}{l}\mathrm{IC}^{2} \\
(+/-)\end{array}$ & $\begin{array}{c}\text { RSD }^{3} \\
(\%)\end{array}$ \\
\hline Óleo essencial (08/97) & $0,00313^{\mathrm{a}}$ & 0,0000100 & 0,0000143 & 0,32 \\
\hline Cera $(08 / 97)$ & $0,00318^{\mathrm{a}}$ & 0,0000173 & 0,0000248 & 0,54 \\
\hline $\begin{array}{l}\text { Óleo do tanque } \\
(06 / 08 / 99)\end{array}$ & $0,00357^{\mathrm{a}}$ & 0,0000387 & 0,0000555 & 1,08 \\
\hline $\begin{array}{c}\text { Óleo da centrífuga } \\
(27 / 08 / 99)\end{array}$ & $0,00335^{\mathrm{a}}$ & 0,0000240 & 0,0000344 & 0,70 \\
\hline $\begin{array}{c}\text { Óleo do arraste a } \\
\text { vapor }(02 / 9 / 99)\end{array}$ & $0,00350^{\mathrm{a}}$ & 0,0000141 & 0,0000202 & 0,40 \\
\hline \multicolumn{5}{|c|}{$\begin{array}{l}{ }^{1} \text { Estimativa do desvio padrão (standard deviation) } \mathrm{SD}=\sqrt{\frac{\left(\mathrm{x}_{\mathrm{i}}-\mathrm{X}\right)^{2}}{\mathrm{n}-1}} ;{ }^{2} \text { Intervalo de confiança }(95 \%), \mathrm{IC}= \pm \frac{\mathrm{t.s}}{\mathrm{n}} \\
\text {; } \\
\text { Estimativa do desvio padrão relativo (relative standard deviation) RSD }(\%)=\mathrm{S} \cdot \frac{100}{\mathrm{x}} ;{ }^{\mathrm{a}} \text { Resultados } \\
\text { significativamente iguais (ANOVA e teste ta } 95 \% \text { de confiança). }\end{array}$} \\
\hline
\end{tabular}

\section{CONCLUSÕES}

Uma nova metodologia analítica foi desenvolvida para analisar cumarinas em amostras de Citrus obtidas da técnica de extração por arraste a vapor e prensagem a frio. A extração por arraste a vapor, empregada com o objetivo de extrair os analitos desejáveis, apresentou resultados semelhantes ao método de extração por prensagem a frio, mostrando-se adequado no estudo destes compostos. Com relação à análise qualitativa, foi constatado que a cumarina (presente nas amostras de óleo e cera) e o psoraleno (presente somente na amostra de cera). A análise quantitativa permitiu verificar que o método do padrão interno apresentou maios repetibilidade e que a concentração de cumarinas presente nas diferentes amostras de óleo foram estatisticamente iguais (após tratamento pelos testes ANOVA e t à $95 \%$ confiança), de acordo com Ribani, 2004.

A extração por arraste a vapor, empregada com o objetivo de extrair os analitos desejáveis, apresentou resultados semelhantes ao método industrial de extração por prensagem a frio, mostrando-se como um método adequado no estudo destes compostos.

As condições de análise (HRGC) mostraram-se adequadas para serem empregadas na análise qualitativa e quantitativa das cumarinas presentes em baixa concentração nas amostras de Citrus.

\section{RESUMO}

As plantas cítricas, em especial a laranja, possuem grande importância para a economia brasileira, com destaque à região Sudeste do país. Os óleos essenciais de Citrus constituem um dos mais importantes grupos de matérias primas para várias indústrias, principalmente as de alimentos, farmacêuticas e de perfumaria. $\mathrm{Na}$ área de alimentos, o óleo essencial de laranja é usado para realçar o "flavor" do suco e exportado para o exterior, mais especificamente para os Estados Unidos (principal consumidor). Neste trabalho desenvolveu-se uma metodologia analítica para identificação e quantificação de cumarinas em derivados de plantas cítricas (óleos, ceras) utilizando-se técnicas cromatográficas tais como cromatografia gasosa de alta resolução com sistema de detecção por ionização de chama (HRGC-FID) e sistema de detecção por espectrometria de massas (HRGC-MS). Os resultados obtidos na análise qualitativa utilizando o sistema HRGC-MS empregando o monitoramento do íon seletivo (SIM) indicaram a presença de cumarina nas amostras de óleo essencial e na amostra de cera foi detectada a presença de cumarina e de psoraleno. As análises quantitativas empregando os métodos do padrão externo, padrão interno e adição de padrão apresentaram bons resultados, em especial o método do padrão interno, o qual apresentou maior repetibilidade.

Palavras-chave: Cumarinas, derivados de citrus, HRGC-MSD

\section{AGRADECIMENTOS}

A CAPES-PICDT, CNPq e FAPESP pelo apoio financeiro, ao Prof. Dr. José Vladimir de Oliveira 
(UFRJ) e o Sr. Wilson Andalécio, pelo fornecimento das amostras.

\section{REFERÊNCIAS}

Simões, C. M. e SPitzer, V. (1999), Óleos essenciais. In: Simões, C. M. O.; Schenckel, E. P.; Gosmman, G.; Mello, J. C. P. Farmacognosia. Da planta ao medicamento. Porto Alegre/ Florianópolis. Ed. UFRGS/UFSC, 387-415.

Kuster, R. M. e Roche, L. M. (2001), Cumarinas, cromonas e xantonas. In: Simões, C. M. O.; Schenckel, E. P.; Gosmman, G. Farmacognosia: $\mathrm{Da}$ planta ao medicamento. Porto Alegre/ Florianópolis. Ed. UFRGS/UFSC, 461-479.

Biavatte, M. W.; Koorich, C. A.; Zucatelli, E.; Martinelli, F. H.; Bresolin, T. B.; Leite, S. N. (2004), Coumarin content and physicochemical profile of Mikania levigata extracts. Zetschrft fur Naturforschung, 59, 178-187.

Tanaka, T. (2005), (Semi-centennial commemoration papers on citrus studies). In: Mattos Junior, D.; Negri, J. D.; Pio, R. M.; Pompeu, J. Citros. Cordeirópolis. Centro APTA Citros Sylvio Moreira. 322-334.

Swingle, W. T. e Reece, P. C. (2005), The botany of Citrus and its wild relative. In: Mattos Junior, D.; Negri, J. D.; Pio, R. M.; Pompeu Junior, J. Citros. Cordeirópolis. Centro APTA Citros Sylvio Moreira. 156-163.

De Miranda, J. A. Caracterização fotofísica de derivados de cumarinas. Dissertação Mestrado em Química - Universidade Federal de Uberlândia, 2009.
Robards, K. e Antolovich , M. (1995), Coumarin and Kaurenoic acid. Analyst, 120-124.

Moshonas , M. G.; Shaw , P. E. (1994). Mikania levigata extracts. Journal of Agricultural and Food Chemistry, 42, 1525-1528.

Eight Peak Index of Mass Spectra. (1986), The Royal Society of Chemistry, London, 2. 246-279.

Vilegas, J. H. Y.; De Marchi, E.; Lanças, F. M. (1997), Determination of coumarin and Kaurenoic acid in Mikania glomerata (guaco) leaves by capillary gas chromatography. Phitochemical Analysis, 8, 74-77.

Loghkin, A. V. e Sckanyan, E. I. (2006). Natural coumarin: methods of isolation and analysis. Pharmaceutical Chemistry Journal. 40, 337-346.

Pathak, M. A. e Fitzpatrick, T. B. (1992), Determination of coumarin in citrus. Journal of Photochemistry and Photobiology. B, 14, 3-22.

Microcal Origin, Version 4. (1996). Microcal Software Inc. New York.

Ribani, M.; Botolli, C. B. G.; Collins, C. H.; Jardim, I. C. S. F.; Melo, L. F. C. (2004), Validaçào em métodos cromatográficos e eletroforéticos. Quimica Nova, 27, 01-10. 